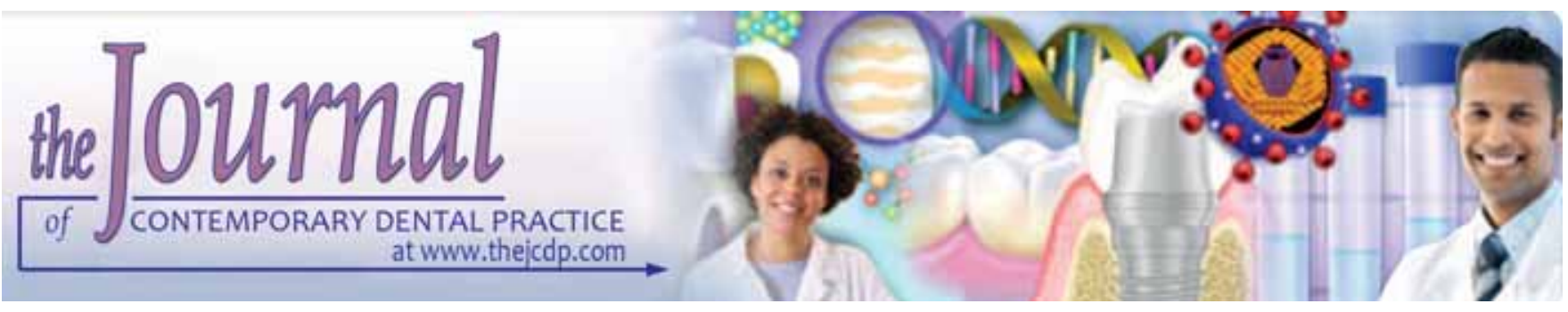

\title{
Serum and Saliva Levels of Cathepsin L in Patients with Acute Coronary Syndrome
}

Iraj Mirzaii-Dizgah, Esmail Riahi

\begin{abstract}
Aim: Coronary artery disease (CAD) is the major cause of death nearly all over the world, and accurate and rapid diagnosis of CAD is of major medical and economic importance. The aim of this study was to evaluate the serum and saliva levels of cathepsin $\mathrm{L}$ in patients with acute coronary syndrome (ACS).
\end{abstract}

Materials and methods: In a cross-sectional study, 39 patients with ACS and 28 with controls were recruited to the study, and cathepsin $L$ levels were measured in serum, resting saliva, and stimulated saliva obtained 12 and $24 \mathrm{~h}$ after the onset of ACS by ELISA method. Statistical analyses of Fisher's exact test, the Student's t-test or Kruskal-Wallis test were performed.

Results: Stimulated saliva cathepsin L levels in patients with ACS 12 hours but not 24 hours after admission showed significant decrease compared with that in control subjects. However, there were no significant differences in serum and unstimulted saliva cathepsin $\mathrm{L}$ levels between groups.

Conclusion: Serum and saliva levels of cathepsin $L$ remain unchanged in patients with ACS and hence may not be a promising factor in CAD risk assessment.

Clinical significance: It seems that serum and saliva cathepsin $\mathrm{L}$ may not be a good biomarker for CHD.

Abbreviations: CAD: Coronary artery disease, ACS: Acute coronary syndrome, CHD: Coronary heart disease, EU: Emergency unit, MI: Myocardial infarction.

Keywords: Cathepsin L, Acute coronary syndrome, Resting saliva, Stimulated saliva.

How to cite this article: Mirzaii-Dizgah I, Riahi E. Serum and Saliva Levels of Cathepsin L in Patients with Acute Coronary Syndrome. J Contemp Dent Pract 2011;12(2):114-119.

Source of support: Nil

Conflict of interest: None declared

\section{INTRODUCTION}

Cathepsins are specific mannose-6-P-rich carbohydrates lysosomal cysteine proteases which they may release into the extracellular milieu in precursor and/or active forms. ${ }^{1-3}$ Cathepsin L is suggested to participate in turnover of intracellular or endocytosed proteins, degradation of extracellular matrix proteins, antigen processing and presentation, bone resorption and various other processes. Most tissues and cells show basal expression of the lysosomal cathepsin L. However, its expression has been found to be increased by inflammatory cytokines including interleukin-6, interleukin-1 and interferon- $\gamma$ as well as several growth factors and oncogenes. ${ }^{4,5}$ Owing to its function in degrading extracellular matrix proteins, cathepsin L has been consistently implicated in atherosclerosis. In this regard, cathepsin L expression has been shown to be increased at intracellular and extracellular spaces in human coronary atheroma lesions ${ }^{6}$ and in carotid atherosclerotic lesions as well. ${ }^{7}$ Moreover, in mouse models of atherosclerosis, deficiency of cathepsin $\mathrm{L}$ gave rise to reduced atherosclerosis, in that it caused smaller atherosclerotic lesions. ${ }^{8}$ Furthermore, cathepsin $\mathrm{L}$ has been found to promote several processes involved in plaque progression, particularly migration of smooth muscle cells and macrophages into the atherosclerotic plaque. ${ }^{9}$

Expression of cathepsin $\mathrm{L}$ was shown to be enhanced in all major cells found in atheroma, including smooth muscle cells, endothelial cells and macrophages, suggesting that it may facilitate vascular wall remodeling. ${ }^{4}$ It contributes to atherogenesis by mediating smooth muscle cell-derived degradation of internal elastica, smooth muscle cells migration and accumulation in the neointima and transmigration of blood-borne leukocytes into the lesion. ${ }^{8}$

Rupture of atherosclerotic plaques is the most common triggering event for formation of occlusive thrombi in arteries and eliciting of acute ischemic events, such as acute coronary syndromes and stroke. Cathepsin $\mathrm{L}$ has been also implicated in plaque rupture. In atherosclerotic lesions, expression of cathepsin L, particularly in macrophages, was associated with formation of a necrotic core and rupture of human carotid plaques. ${ }^{10}$

It has been also found that serum cathepsin L levels increase in patients with coronary artery stenosis, so that, 
the degree of stenosis of the left anterior descending coronary artery correlates with serum cathepsin L levels in these patients. ${ }^{4,11}$ In addition, patients with coronary heart disease (CHD) were found to have increased serum cathepsin L levels even after adjusting for major CHD confounders. Furthermore, serum cathepsin L levels in CHD patients positively correlate with Gensini score, suggesting that serum cathepsin L may reflect the severity of coronary atherosclerotic stenosis. On the basis of these observations, it has been proposed that serum cathepsin L concentrations may have the potential to serve as an independent predictor of human CHD. ${ }^{11}$

Since human studies on serum cathepsin L in CHD are lacking and to further elucidate cathepsin $\mathrm{L}$ alterations in bodily fluids of patients with CHD, we carried out a study on serum and saliva levels of cathepsin $\mathrm{L}$ in patients with acute coronary syndrome. Herein, we report that cathepsin $\mathrm{L}$ concentrations showed significant increases neither in serum nor in saliva of patients with acute coronary syndrome.

\section{MATERIALS AND METHODS}

\section{Study Design}

In a case-control study, 39 consecutive patients who were admitted to the emergency unit (EU) of Imam Hussein Medical Center with typical ischemic chest pains were recruited to this study. Twelve lead electrocardiography and laboratory analysis of serum biomarkers of cardiac necrosis, including creatine kinase, creatine kinase MB isoenzyme and cardiac troponin were performed for all patients. With the aid of these paraclinical tools, a diagnosis of acute coronary syndrome was made for all of these patients by an expert cardiologist. Of these patients, 10 were diagnosed as having unstable angina and 29 were identified as patients with acute ST-elevation MI. In addition, 28 subjects who were admitted to the ophthalmic ward of Imam Hussein Hospital with the purpose of having an eye surgery, and with no history of coronary heart disease, were recruited to the study as control subjects.

Baseline clinical characteristics were collected for all participants via a questionnaire with a set of inquiries about their demographic information and clinical history, including recognized diseases, medical interventions, medications and other health problems.

Several factors have been postulated to affect cathepsin L levels, including cancer, arthritis, renal failure, chronic hepatic diseases, hypothyroidism, fever or inflammatory symptoms, diabetes, parasitosis, tuberculosis, bacterial/viral infection, bone fracture, or receiving heparin, antibiotics, hormone, aspirin, angiotensin-converting enzyme inhibitors (ACEIs), angiotensin receptor blockers (ARBs), or statins. ${ }^{4,11}$ We dealt with most of these confounders as exclusion criteria for this study, so that patients with any of these factors, except for diabetes and taking NSAIDs, ACEIs, ARBs and statins, were not selected for this study. However, matching of the cases and controls was performed in a way that there was no significant difference in terms of the excepted factors between these groups.

\section{Saliva and Serum Sampling}

Saliva and blood samples were obtained from patients with acute MI about 12 and 24 hours after presentation to emergency unit (EU) and from patients with unstable angina and control subjects when they were hospitalized in cardiac care unit or ophthalmic ward, respectively. All participants were first informed about the sampling procedure. They were requested not to eat, drink, smoke cigarette or brush their teeth for 1 hour before saliva collection. For resting saliva sampling, subjects rinsed their mouths with tap water, and 2 minutes later swallowed all their oral fluid. Thereafter, they spitted 2 to $3 \mathrm{ml}$ of their resting whole saliva in a graded plastic tube with no active movement of mouth wall or sucking the oral cavity. Saliva gathering was carried on for 5 minutes unless 2 to $3 \mathrm{ml}$ of saliva had been collected.

Following resting saliva collection, subjects were asked to Chew a piece of natural neutral gum with a given size for 2 minutes. After that, subjects either spat all the oral fluid out or entirely swallowed it, and thereafter they began to collect the stimulated whole saliva into another graded tube while continuing chewing the gum.

The timing of saliva gathering was recorded in order to estimate saliva flow rate and salivary output of cathepsin L. Salivary output of cathepsin L (ng/min) was calculated as saliva flow rate $(\mathrm{ml} / \mathrm{min})$ multiplied by salivary concentration of cathepsin L (ng/ml). Two milliliters of venous blood were drawn immediately after saliva sampling. Following sample collection, the specimens were centrifuged at $3800 \mathrm{gm}$ for 10 minutes, and then the serum and saliva supernatants were isolated and divided into aliquots. The aliquots were stored in $-70^{\circ} \mathrm{C}$ for later analysis of cathepsin L.

\section{Laboratory Measurements}

Enzyme-linked immunosorbent assay (ELISA) was applied to measure the plasma concentrations of cathepsin L. Human cathepsin L ELISA kit was provided from BioVendor company. Determination of cathepsin L levels was carried out according to the manufacturers' instructions. Briefly, after preparing all needed buffers and reagents, serum and saliva samples were added to microwells coated with first antibody, and then biotin-conjugated antihuman cathepsin L antibody was added and allowed to bind to human 
cathepsin L captured by the first antibody for 2 hours of incubation. Then, the wells were washed 3 times with wash buffer using a plate washer to remove unreacted proteins. Next, streptavidin-HRP was added to the wells and allowed to bind to the biotin-conjugated antibody for 1 hour of incubation, followed by washing the plates. After that, TMB substrate solution was added to the wells and color development proceeded for 15 minutes, when the reaction was ended by adding stop solution. Subsequently, absorbance was read at a wavelength of $450 \mathrm{~nm}$ on an ELISA reader and cathepsin $L$ concentrations were determined, with the limit of detection being $1.71 \mathrm{ng} / \mathrm{ml}$. The intra- and interassay coefficients of variation were 6.2 and $6 \%$ respectively for the cathepsin $\mathrm{L}$ assay.

Serum levels of CK, CK-mb and troponin I, triglycerides, total cholesterol, LDL cholesterol and HDL cholesterol were measured as part of ACS patients' routine clinical management protocol using standard clinical chemistry procedures.

\section{STATISTICAL ANALYSIS}

Assessment of distribution for all continuous variables was made in the first instance. For analyzing goodness of normal fitting, Shapiro-Wilk W test was applied to check if the data has a normal distribution. In descriptive statistics, results of normally distributed continuous variables were presented as mean \pm standard deviation (SD) and of continuous variables with non-normal distribution were expressed as median and interquartile range. Nominal data was shown as frequencies. Detection of between-groups differences was carried out with Fisher's exact test, independent samples t-test or Kruskal-Wallis test, as appropriate. To evaluate correlations between numerical variables, Mahalanobis distance test was first used for outlier analysis and following exclusion of outlying observations, the Spearman rho test was applied to determine association between variables. A p-value $(<0.05)$ was considered to be statistically significant.

\section{RESULTS}

Basal clinical properties of participants were summarized in Table 1. None of the characteristics mentioned in the Table 1 displayed significant difference between study groups, except for hypertension which showed merely a marginal significance $(p=0.08)$.

Serum and saliva cathepsin L had skewed distribution; hence, we utilized nonparametric tests to compare study groups. Values from control subjects were compared with those of ACS patients, including patients with acute $\mathrm{MI}$ and patients with unstable angina, from whom samples were obtained 12 and/or 24 hours after admission to EU.

Serum cathepsin L levels in patients with ACS, both 12 and 24 hours after admission to EU showed no significant difference with those in control subjects ( $p>0.05)$ (Fig. 1).

Regarding resting saliva levels of cathepsin $\mathrm{L}$, there was also no significant difference between any of the study groups ( $p>0.05)$. Even when saliva flow rates were taken into consideration, saliva cathepsin L did not significantly differ between groups ( $p=0.19$ ) (Fig. 2A).

Stimulated saliva cathepsin L concentration in patients with ACS 12 hours after admission showed significant decrease compared with that in control subjects ( $\mathrm{p}=0.0003$ ). But, cathepsin L concentration in stimulated saliva obtained from ACS patients 24 hours after admittance was not significantly different from that of healthy controls. Likewise, stimulated saliva cathepsin L output in ACS patients 12 hours, but not 24 hours after admission displayed significant decrease compared with that in healthy controls ( $\mathrm{p}=0.02)$ (Figs 2B).

Serum cathepsin L showed partial, but statistically significant, correlation with resting saliva cathepsin L output (rho $=0.24, \mathrm{p}=0.02$ ). However, it was not correlated with other salivary factors. Serum cathepsin L had significant correlation with LDL cholesterol in ACS patients (rho $=0.39, \mathrm{p}=0.01$ ).

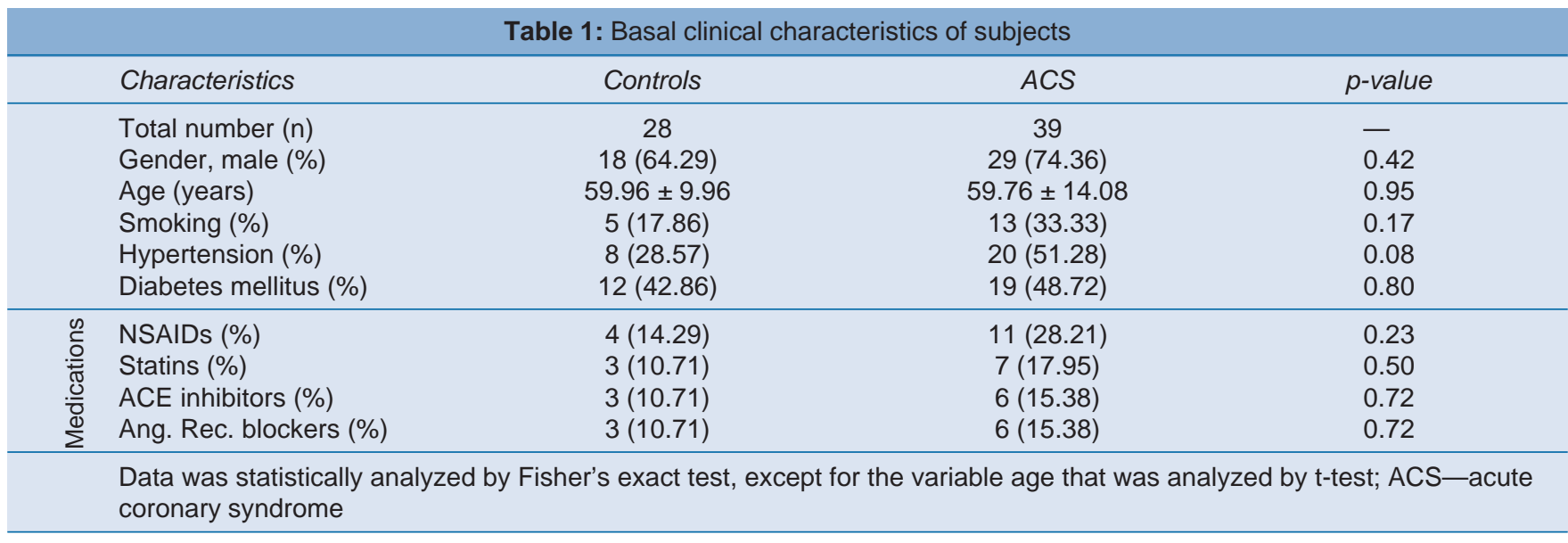




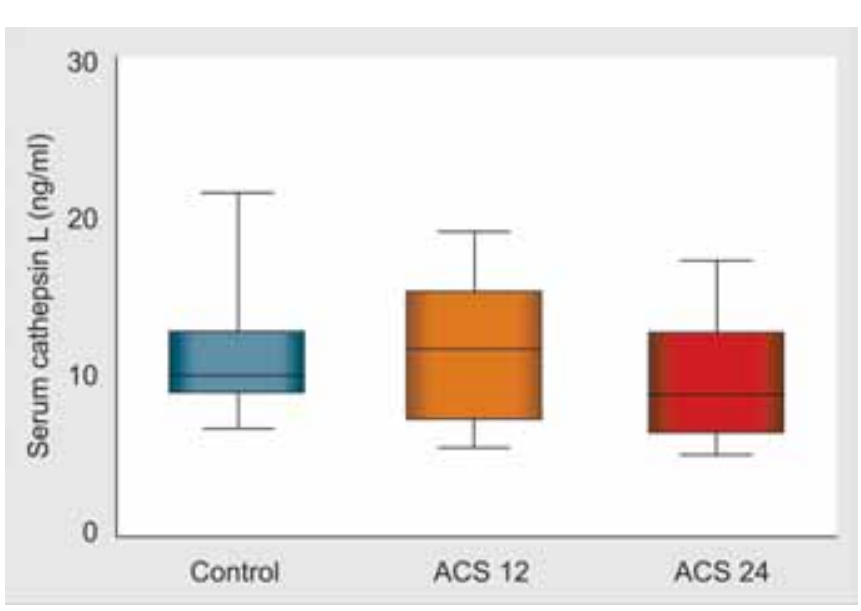

Fig. 1: Variability chart showing the distribution of serum cathepsin $L$ concentrations in each group. There was no significant difference between study groups. The ends of the box are the 25th and 75th quartiles. The difference between the quartiles is the interquartile range. The line across the middle of the box identifies the median sample value. The lines extended from the ends of each box, which are called whiskers, identify the 10 to 90 percentiles
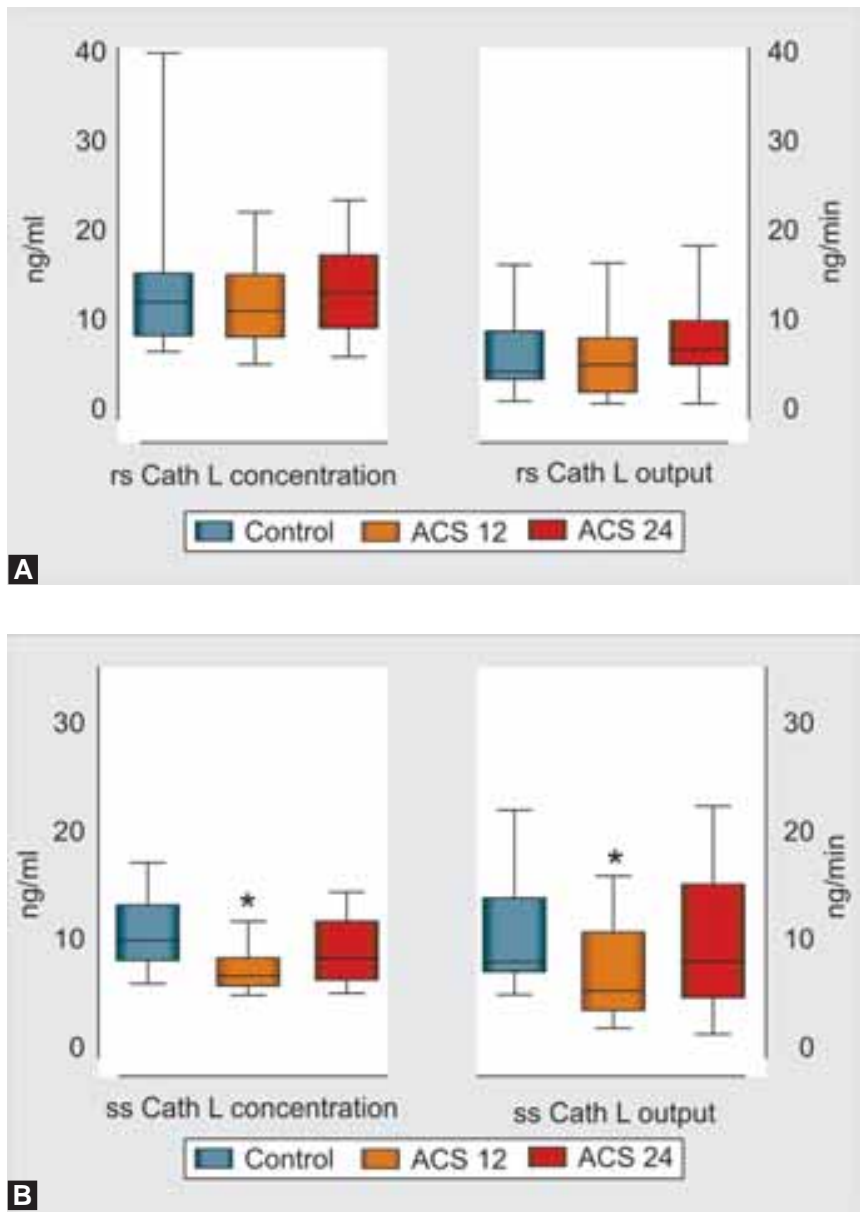

Figs 2A and B: Variability chart demonstrating the distribution of cathepsin $L$ concentrations (left) and outputs (right) at resting (a) and stimulated (b) saliva flow rates. (a) There was no significant difference between study groups. (b) Patients with ACS 12 hours after admittance had reduced levels and output of stimulated saliva cathepsin L compared to control subjects. Asterisks indicate significant differences with corresponding control group

\section{DISCUSSION}

The key finding of this study was that serum cathepsin L concentrations did not differ between patients with ACS and people with no documented coronary heart disease. Moreover, saliva levels and also outputs of cathepsin L at resting saliva flow rates did not show any difference between ACS patients and apparently control group. However, saliva levels and outputs of cathepsin L at stimulated saliva flow rates found to be reduced in ACS patients 12 hours after onset of ischemic heart attack.

Several lines of evidence have consistently highlighted the role of cysteine protease cathepsin L activity in vascular wall remodeling during the lifelong process of atherosclerosis. Cathepsin L has been postulated to impair coronary endothelial function via production of a collagen XVIIIderived antiangiogenesis factor, endostatin, which in turn enhances $\mathrm{O}_{2}^{-}$production through activation of $\mathrm{NAD}(\mathrm{P}) \mathrm{H}$ oxidase. ${ }^{12}$ It takes part in the formation and progression of atherosclerotic plaques and may also participate into the destabilization of advanced plaques. ${ }^{9}$

Limited attempts have been recently made to associate serum levels of cathepsin $L$ with some special conditions, such as pre-eclampsia, ${ }^{13}$ obesity, ${ }^{14}$ coronary artery stenosis, ${ }^{4}$ CHD,${ }^{11}$ coronary artery ectasia ${ }^{15}$ and coronary collateral formation. ${ }^{16}$ Among these, the connection between coronary artery disease and serum cathepsin $\mathrm{L}$ is of major importance, in that it is relevant to the results of our study. In 2009, Yingxian Liu et al showed that patients with CHD had elevated levels of serum cathepsin L in comparison to patients without CHD. The beauty of their study was the state-dependent increases in serum cathepsin L concentrations - the more severe the disease, the higher the levels of serum cathepsin L. Patients with stable angina had higher levels of cathepsin L compared with those without CHD, patients with unstable angina had more cathepsin L levels than those with stable angina, patients with acute or old MI showed higher levels of cathepsin $L$ than those with unstable angina, and finally serum levels of cathepsin L remained elevated in patients with old MI. ${ }^{11}$

The results obtained from our study are not consistent with those of Liu's study, as we found no significant alterations in serum cathepsin L concentrations of patients with ACS compared with those of subjects without CHD. Levels of cathepsin L in serum of ACS patients were measured twice, 12 and 24 hours after onset of ischemic events, none of them displayed higher values relative to those of controls. At the present time, we cannot offer any explanation for this discrepancy other than having different methodologies. 
In another set of our survey, we evaluated possible changes of cathepsin L in saliva obtained from study subjects. Saliva levels and outputs of cathepsin $\mathrm{L}$ at resting saliva flow rates showed no significant difference between ACS patients and people without CHD. This result is similar to the result acquired from serum assay of cathepsin L. Regardless of not being elevated in ACS patients, cathepsin $\mathrm{L}$ has been found, for the first time, to be measurable in salivary medium.

The results of cathepsin L assays in stimulated saliva of study groups are quite similar to those of serum and resting saliva, except that cathepsin L levels and outputs in stimulated saliva of ACS patients 12 hours after admittance were less than those of controls. This observation, however, seems to be of no importance regarding evaluating risk of CHD.

We found merely a poor correlation between serum concentration of cathepsin $\mathrm{L}$ and resting saliva output of this biochemical. Moreover, serum cathepsin L was not associated with other salivary parameters. This might be due to the ubiquitous expression of cathepsin L. If the serum cathepsin L was the only source of salivary cathepsin L, it would be expected to find a close connection between serum and saliva values of this peptide. It is possible that cathepsin $\mathrm{L}$ is produced by salivary gland and released into oral fluids. In support of this claim, it has been shown that cathepsin $\mathrm{L}$ can be produced in human gingival fibroblasts, and its production was enhanced under the influence of interleukin-6, implicating inflammatory processes in expression of cathepsin L. ${ }^{17}$ Furthermore, saliva levels of cathepsin L were found to be generally more than its serum concentrations (data not shown). Owing to its peptide structure, cathepsin L seems unlikely to be actively transported from plasma and accumulated into salivary secretions. This observation, hence, is in favor of salivary production and secretion of cathepsin L.

The rationale of our study for measuring cathepsin $\mathrm{L}$ in salivary secretions is that saliva is being considered as a diagnostic fluid of the future. Saliva is believed to be a mirror of the body and may be acknowledged as a promising medium for monitoring health and disease states of an individual in healthcare programs. Several lines of evidence have consistently validated and proposed using salivary assays for diagnosing, monitoring or predicting prognosis of diseases. In this regard, it has been shown that several biochemical molecules can be measured in oral fluids of diseased patients, for example, steroid hormones, such as cortisol, ${ }^{18}$ progesterone ${ }^{19}$ and $17 \beta$-estradiol; ${ }^{20}$ protein/ polypeptide hormones, such as CA15- $3,{ }^{21} \mathrm{CA} 125,{ }^{22}$ creatine kinase $\mathrm{MB},{ }^{23}$ creatine phosphokinase ${ }^{24}$ and parathyroid hormone. ${ }^{25}$ Much of the attention saliva receives as a biological specimen is due to the quick, uncomplicated and noninvasive nature of sample collection. ${ }^{26}$ Furthermore, oral fluid sampling is safe for both the operator and the patient and has easy and low-cost storage. To establish saliva as an alternative medium to plasma for various biological assays, there must be a high correlation between plasma and saliva levels of measured parameters. ${ }^{23}$ The lack of such a close association between serum and saliva measures of cathepsin L implies that salivary cathepsin L may not be a good alternative for being used as a surrogate for serum cathepsin L in diagnostic assays, screening programs or epidemiological studies.

\section{CONCLUSION}

In conclusion, the results of our study indicate that serum and saliva levels of cathepsin $\mathrm{L}$ remain unchanged in patients with acute coronary syndrome and hence may not be a promising factor in CHD-risk assessment. It seems that more investigations need to be done to deal with the controversy raised by this study about the previously proposed value of serum cathepsin L as a biomarker for CHD.

\section{CLINICAL SIGNIFICANCE}

It seems that serum and saliva cathepsin L may not be a good biomarker for CHD.

\section{REFERENCES}

1. Tang Q, Cai J, Shen D, Bian Z, Yan L, Wang YX, et al. Lysosomal cysteine peptidase cathepsin L protects against cardiac hypertrophy through blocking AKT/GSK3 beta signaling. J Mol Med 2009;87:249-60.

2. Strojnik T, Kavalar R, Trinkaus M, Lah TT. Cathepsin L in glioma progression: Comparison with cathepsin B. Cancer Detect Prev 2005;29:448-55.

3. Hook V, Yasothornsrikul S, Greenbaum D, Medzihradszky KF, Troutner K, Toneff T, et al. Arg/Lys aminopeptidase: A distinct prohormone processing pathway for the biosynthesis of peptide neurotransmitters and hormones. Biol Chem 2004;385:473-80.

4. Liu J, Sukhova GK, Yang JT, Sun J, Ma L, Ren A, et al. Cathepsin $\mathrm{L}$ expression and regulation in human abdominal aortic aneurysm, atherosclerosis and vascular cells. Atherosclerosis 2006;184: 302-11.

5. Ravanko K, Jarvinen K, Helin J, Kalkkinen N, Holtta E. Cysteine cathepsins are central contributors of invasion by cultured adenosylmethionine decarboxylase-transformed rodent fibroblasts. Cancer Res 2004;64:8831-38.

6. Li W, Dalen H, Eaton JW, Yuan XM. Apoptotic death of inflammatory cells in human atheroma. Arterioscler Thromb Vasc Biol 2001;21:1124-30.

7. Mattock KL, Gough PJ, Humphries J, Burnand K, Patel L, Suckling KE, et al. Legumain and cathepsin-L expression in human unstable carotid plaque. Atherosclerosis 208:83-89.

8. Kitamoto S, Sukhova GK, Sun J, Yang M, Libby P, Love V, et al. Cathepsin L deficiency reduces diet-induced atherosclerosis in low-density lipoprotein receptor-knockout mice. Circulation 2007;115:2065-75. 
9. Lafarge JC, Naour N, Clement K, Guerre-Millo M. Cathepsins and cystatin $\mathrm{C}$ in atherosclerosis and obesity. Biochimie 92:1580-86.

10. Li W, Kornmark L, Jonasson L, Forssell C, Yuan XM. Cathepsin $\mathrm{L}$ is significantly associated with apoptosis and plaque destabilization in human atherosclerosis. Atherosclerosis 2009;202:92-102.

11. Liu Y, Li X, Peng D, Tan Z, Liu H, Qing Y, et al. Usefulness of serum cathepsin $\mathrm{L}$ as an independent biomarker in patients with coronary heart disease. Am J Cardiol 2009;103: 476-81.

12. Zhang F, Zhang Y, Li PL. Dependence of cathepsin L-induced coronary endothelial dysfunction upon activation of $\mathrm{NAD}(\mathrm{P}) \mathrm{H}$ oxidase. Microvasc Res 2009;78:45-50.

13. Dong $M$, Wang $H$, Huang $H$. Alterations of serum cathepsins $B$ and L in pre-eclampsia. Clin Chim Acta 2007;377:285-87.

14. Naour N, Rouault C, Fellahi S, Lavoie ME, Poitou C, Keophiphath M, et al. Cathepsins in human obesity: Changes in energy balance predominantly affect cathepsins in adipose tissue and in circulation. J Clin Endocrinol Metab 2010;95:1861-68.

15. Zografos TA, Haliassos A, Korovesis S, Giazitzoglou E, Serelis J, Katritsis DG. Serum cathepsin levels in coronary artery ectasia. Int J Cardiol 145:606-07.

16. Zhang J, Wang P, Huang YB, Li J, Zhu J, Luo X, et al. Plasma cathepsin $\mathrm{L}$ and its related pro/antiangiogenic factors play useful roles in predicting rich coronary collaterals in patients with coronary heart disease. J Int Med Res 38:1389-1403.

17. Yamaguchi T, Naruishi K, Arai H, Nishimura F, Takashiba S. IL-6/sIL-6R enhances cathepsin B and L production via caveolin-1-mediated JNK-AP-1 pathway in human gingival fibroblasts. J Cell Physiol 2008;217:423-32.

18. Agha-Hosseini F, Mirzaii-Dizgah I, Mirjalili N. Relationship of unstimulated whole saliva cortisol level with severity of oral dryness feeling in menopausal women. Australian Dental Journal 2011;56:171-74.

19. Mirzaii-Dizgah I, Agha-Hosseini F. Stimulated and unstimulated saliva progesterone in menopausal women with oral dryness feeling. Clin Oral Investig 2011.
20. Agha-Hosseini F, Mirzaii-Dizgah I, Mansourian A, Khayamzadeh M. Relationship of stimulated saliva 17 beta-estradiol and oral dryness feeling in menopause. Maturitas 2009;62(2):197-99.

21. Agha-Hosseini F, Mirzaii-Dizgah I, Rahimi A. Correlation of serum and salivary CA15-3 levels in patients with breast cancer. Med Oral Patol Oral Cir Bucal 2009;4(10):e521-e24.

22. Agha-Hosseini F, Mirzaii-Dizgah I, Rahimi A, SeilanianToosi M. Correlation of serum and salivary CA125 levels in patients with breast cancer. J Contemp Dent Pract 2009;10(6): E001-E008.

23. Mirzaii-Dizgah I, Hejazi SF, Riahi E, Salehi MM. Saliva-based creatine kinase MB measurement as a potential point-of-care testing for detection of myocardial infarction. Clin Oral Invest 2011.

24. Mirzaii-Dizgah I, Jafari-Sabet M. Unstimulated whole saliva creatine phosphokinase in acute myocardial infarction. Oral Dis.

25. Agha-Hosseini F, Mirzaii-Dizgah I, Mansourian A, ZabihiAkhtechi G. Serum and stimulated whole saliva parathyroid hormone in menopausal women with oral dry feeling. Oral Surg Oral Med Oral Pathol Oral Radiol Endod 2009;107(6):806-10.

26. Agha-Hosseini F, Dizgah IM, Amirkhani S. The composition of unstimulated whole saliva of healthy dental students. J Contemp Dent Pract May 1, 2006;7(2):104-11.

\section{ABOUT THE AUTHORS}

\section{Iraj Mirzaii-Dizgah}

(Corresponding Author)

Iraj Mirzaii-Dizgah, Professor, Department of Physiology AJA University of Medical Sciences, Tehran, Iran, e-mail: emirzaii@razi.tums.ac.ir

\section{Esmail Riahi}

Researcher, Department of Physiology, AJA University of Medical Sciences, Tehran, Iran 